\title{
Accurate Measurement of In-Band FWM Power in DWDM Systems Over Nonzero Dispersion Fibers
}

\author{
Antonella Bogoni, Luca Potì, Member, IEEE, and Alberto Bononi
}

\begin{abstract}
A novel accurate measurement technique of the in-band four-wave mixing (FWM) power in dense wavelength-division multiplexing (DWDM) over nonzero dispersion fibers is presented. By measuring only two out-of-band FWM terms generated by three fixed channels, the FWM efficiencies on all DWDM channels can be evaluated and the in-band signal-FWM crosstalk ratio can be calculated. A comparison with other known methods proves the better accuracy of the proposed technique.
\end{abstract}

Index Terms-Dense wavelength-division multiplexing (DWDM), fiber-optic transmission systems, four-wave mixing (FWM).

\section{INTRODUCTION}

$\mathbf{T}$ HE PERFORMANCE of dense wavelength-division-multiplexing (DWDM) transmission systems over low dispersion fibers is mostly degraded by four-wave mixing (FWM) [1] so that an accurate evaluation of the in-band crosstalk FWM terms is mandatory for system design. Among the most popular experimental techniques, the suppression of the channel in the band where the FWM power is to be measured (channel suppression (CS) method) [2] is not accurate, since some important FWM terms are also suppressed. It is also not accurate to evaluate the FWM power by slightly detuning the channel in the band of interest (channel detuning (CD) method) [1, Fig. 2], since the efficiency of the FWM terms is altered.

In this letter, we present a novel method for the accurate evaluation of the in-band FWM in DWDM equally spaced systems over nonzero dispersion fibers (NZDFs). In the measurement, all DWDM channels are suppressed except for three suitably chosen test channels, which are used to take only two FWM efficiency measurements. From such measurements, one calculates the in-band FWM power when all DWDM channels are active. We call such method the three-channel (TC) method. A comparison with the commonly used CS and CD methods shows the higher accuracy of the novel method. The TC method is experimentally verified on a four-channel WDM system.

\section{THEORY}

The FWM power $P_{i j k}$, generated by three polarization-aligned continuous-wave (CW) channels at frequencies $f_{i}$, $f_{j}$, and $f_{k}$, is [1]

$$
P_{i j k}=d^{2} P_{i} P_{j} P_{k} b \eta_{i j k}
$$

Manuscript received August 2, 2002; revised October 22, 2002.

A. Bogoni is with CNIT, 56124 Pisa, Italy and also with Parma University, Parco Area delle Scienze, 43100 Parma, Italy.

L. Potì is with CNIT, 56124 Pisa, Italy.

A. Bononi is with Parma University, Parco Area delle Scienze, 43100 Parma, Italy.

Digital Object Identifier 10.1109/LPT.2002.806853 where $d$ is the degeneracy factor, which takes value 1 and 2 for degenerate and nondegenerate terms, respectively, and

$$
\begin{aligned}
b & =\left(\frac{2 \pi n_{2}}{A_{\mathrm{eff}} \lambda_{c}}\right)^{2} e^{-\alpha z} L_{\mathrm{eff}}^{2} \\
\eta_{i j k} & =\frac{\alpha^{2}}{\alpha^{2}+\Delta \beta_{i j k}^{2}}
\end{aligned}
$$

where $n_{2}$ is the nonlinear coefficient, $\lambda_{c}$ the central wavelength, $A_{\text {eff }}$ the core effective area, $L_{\text {eff }}$ the effective length, $\alpha$ the fiber attenuation, $z$ the fiber length, and $P_{i}, P_{j}$, and $P_{k}$ the input power of channels $\mathrm{i}, \mathrm{j}$, and $\mathrm{k}$. The expression of the efficiency $\eta_{i j k}$ is valid for $z$ much longer than $1 / \alpha$, and the phase matching coefficient $\Delta \beta_{i j k}$, away from the zero dispersion wavelength (ZDW) $\left(\left|D_{\mathrm{c}}\right|>1 \mathrm{ps} / \mathrm{nm} / \mathrm{km}\right)$, is given by [1]

$$
\Delta \beta_{i j k}=\frac{2 \pi c}{\lambda_{c}^{2}}\left(\lambda_{i}-\lambda_{k}\right)\left(\lambda_{j}-\lambda_{k}\right) \cdot D_{c}
$$

where $c$ is the light speed, $D_{c}$ is the fiber dispersion, and $\Delta \lambda_{\mathrm{ik}}$ and $\Delta \lambda_{\mathrm{jk}}$ are the wavelength spacings between channels $i, k$ and $j, k$. Note that, away from the ZDW, (1)-(3) accurately predict FWM power, even when the ZDW is not constant along the fiber [3].

In the case of channels arranged on an equally spaced grid of resolution $\Delta \lambda, \Delta \beta_{i j k}$ takes the discrete values

$$
\Delta \beta_{n}=n\left(\frac{2 \pi c}{\lambda_{c}^{2}}\right) D_{c} \Delta \lambda^{2} \quad n=(i-k)(j-k) .
$$

In Fig. 1, we summarize all FWM terms falling on each frequency in an equally spaced four-channel system. Each term is represented by the indices $i j k$ of the three channels involved in the combination. For instance, the term 123 labels the FWM term jointly generated by channels 1,3 , and 2 . Note that since this term falls on channel 2, its power cannot be measured by simply suppressing channel 2 , and this is the main limitation of the CS method.

For each FWM term, the corresponding phase-matching coefficient $\Delta \beta_{\mathrm{n}}$ is also shown in Fig. 1. Some FWM terms, at different frequencies, may have the same $\Delta \beta_{\mathrm{n}}$ values, and thus the same efficiency values.

In Fig. 2 the FWM efficiency $\eta_{i j k}$ is plotted versus the discrete values of $\Delta \beta_{\mathrm{n}}$ in the case of a nonzero dispersion-shifted fiber (NZDSF) with $D_{c}=2 \mathrm{ps} / \mathrm{nm} / \mathrm{km}$ and $\Delta \lambda=0.4 \mathrm{~nm}$. The efficiency also takes on quantized values $\eta\left(\Delta \beta_{\mathrm{n}}\right)$, with $n$ representing the order of quantized efficiency. Now, from (1) and (2) it is clear that all FWM powers are immediately calculated when the discrete efficiency coefficients

$$
\eta_{n}=b \frac{\alpha^{2}}{\alpha^{2}+\Delta \beta_{n}^{2}}=b \frac{\alpha^{2}}{\alpha^{2}+n^{2} \Delta \beta_{1}^{2}}
$$




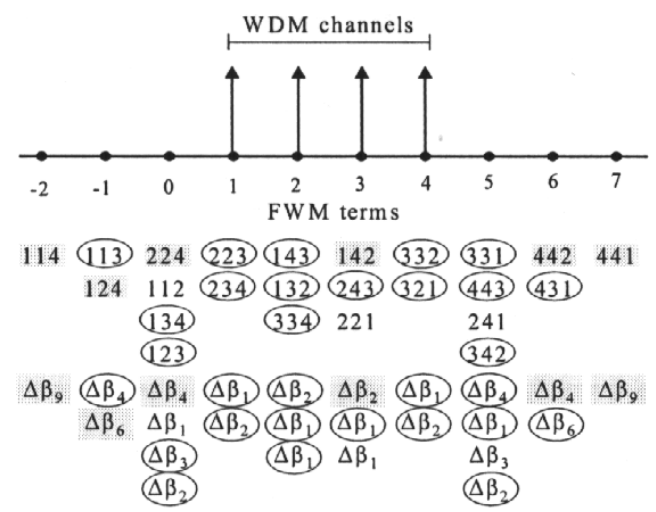

Fig. 1. FWM contributions and corresponding $\Delta \beta_{n}$ for a four-channel system.

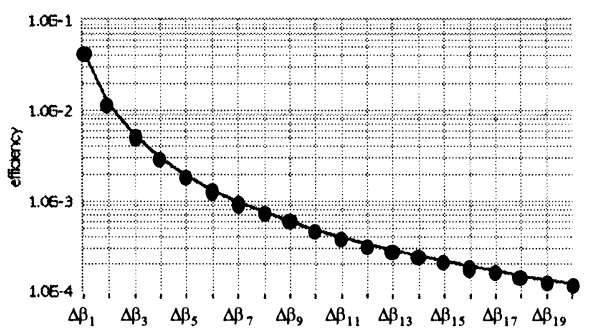

Fig. 2. FWM efficiency versus phase matching terms.

are known. If we can reliably measure such coefficients for two quantized efficiency orders $i$ and $j$, with $i<j$, then for all $n$ the efficiency coefficients can be calculated as

$$
\eta_{n}=\frac{\left(j^{2}-i^{2}\right) \eta_{i} \eta_{j}}{\left(n^{2}-i^{2}\right) \eta_{i}-\left(n^{2}-j^{2}\right) \eta_{j}}
$$

and we do not need any precise knowledge of the fiber parameters. Since such coefficients are decreasing in $n$, as seen in Fig. 2, we should use the lowest possible values for $i$ and $j$ in (6), as these correspond to the largest ones, those that can be most reliably measured.

In order to measure the efficiency term $\eta_{1}$, consider again Fig. 1. When channels 3 and 4 are switched OFF, all FWM terms containing the indices 3, 4 disappear (circled and gray terms in the figure). Hence, the power of the degenerate FWM term 112 (or 221) falling on channel 0 (or 3) can be measured, and we get

$$
\eta_{1}=\frac{P_{112}}{P_{1}^{2} P_{2}} .
$$

Similarly, we would like to measure the second largest coefficient $\eta_{2}$. With channels 1,2 , and 4 ON and channel 3 OFF, all FWM terms containing the index 3 in Fig. 1 (circled terms) disappear. It is clear that the FWM term of index 2 always appears together with another degenerate term, and no combination of three or more active channels exists that allows a direct measure of the $\eta_{2}$ term.

However, we can easily get the efficiency term $\eta_{3}$ by measuring the nondegenerate FWM term 241 on channel 5 as

$$
n_{3}=\frac{P_{241}}{4 P_{2} P_{4} P_{1}} \text {. }
$$

Once $\eta_{1}$ and $\eta_{3}$ have been measured, we can use (6) with $i=1$ and $j=3$ to compute all remaining efficiency coefficients. If

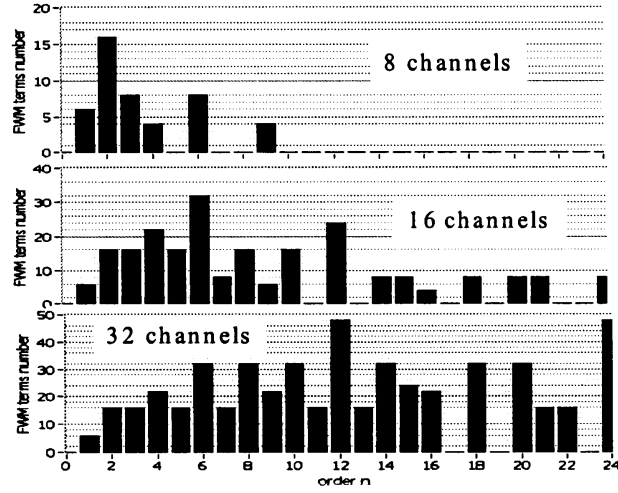

Fig. 3. Number of FWM terms falling in the central channel band for an 8-, 16-, and 32-channel system versus the efficiency order $n$.

we consider equal channel powers, we obtain the total in-band FWM power on channel $f$ as

$$
P_{f_{\mathrm{FWM}}}=P^{3} \sum_{n} C_{n} \eta_{n}
$$

where $C_{n}$ is the number of FWM terms with order $n$ falling in the band of channel $f$. Note that each nondegenerate term must be counted four times.

Fig. 3 shows the value of the $C_{n}$ coefficients versus the order $n$ for three different systems, with $f$ being the central (worst case) channel of the comb.

In the general case, the computation time of all FWM terms grows exponentially with the number of channels N. For instance, it took 20 s for our software to run on a Pentium III PC when $\mathrm{N}=512$, and $130 \mathrm{~s}$ for $\mathrm{N}=1024$.

However, the contributions to the total FWM power at a fixed frequency rapidly decrease to zero when the order $n$ gets large, as seen in Fig. 2, so that the summation in (9) can be safely truncated to a highest order $n_{\max }$, which depends on chromatic dispersion and channel spacing values. For instance, if we consider a NZDSF, and $\Delta \lambda=0.4 \mathrm{~nm}$, by inserting the $\eta_{1}$ and $\eta_{2}$ values obtained from Fig. 2 in (6), we find that $\eta_{24}<\eta_{1} / 10^{4}$.

\section{COMPARISON With OTHER MethodS}

The newly proposed procedure has been compared with the CS and CD methods. First note that the new technique allows for the determination of the FWM crosstalk for all channels by measuring only two isolated FWM terms. Instead, the other techniques need as many measures as the number of transmitted channels. To complete the comparison, we define a relative error in the measure of FWM crosstalk in the channel $f$-band as

$$
\varepsilon=\frac{P_{f_{\mathrm{FWM}}}-P_{f_{\mathrm{FWM}}}^{M}}{P_{f_{\mathrm{FWM}}}}
$$

where $P_{f \mathrm{FWM}}$ is the actual FWM power in the channel band and $P_{f \mathrm{FWM}}^{M}$ is the measured FWM power. When the channel $f$ is suppressed, the measured in-band FWM power in the CS method is $\mathrm{P}_{f \mathrm{FWM} C S}^{M}=\Sigma P_{i j k}$ with $i, j$, and $k$ not coinciding with $f$. If we detune the channel $f$, the true FWM efficiency $\eta$ of the terms generated by itself changes to $\eta^{\prime}$, therefore the measured in-band FWM power in the CD method is $P_{f \mathrm{FWM} \mathrm{CD}}^{M}=$ $\mathrm{P}_{f \mathrm{FWM} \mathrm{CS}}^{M}+\Sigma P_{i j k}\left(\eta^{\prime}\right)$ with $i$ or $j$ or $k$ coinciding with $f$. The error introduced by the TC method is due to the dispersion slope, 


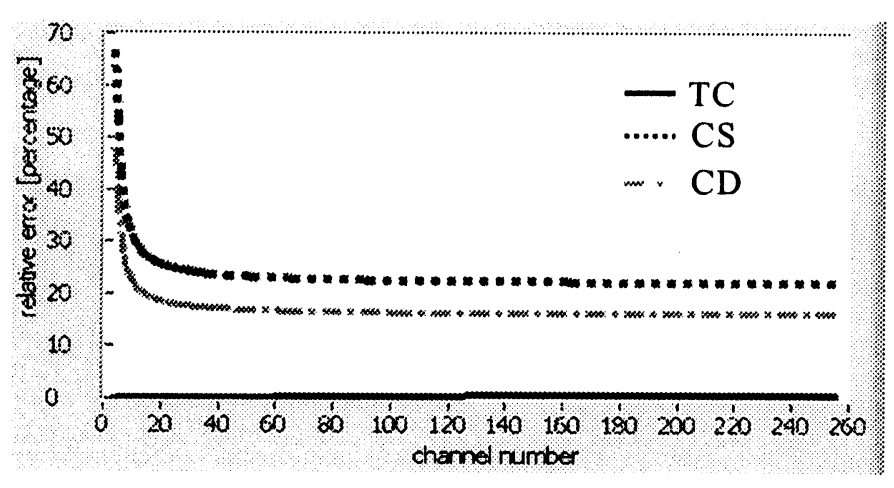

Fig. 4. Relative error $\varepsilon$ obtained with three different methods for the central WDM channel versus the number of WDM channels in the case of NZDSF fiber.

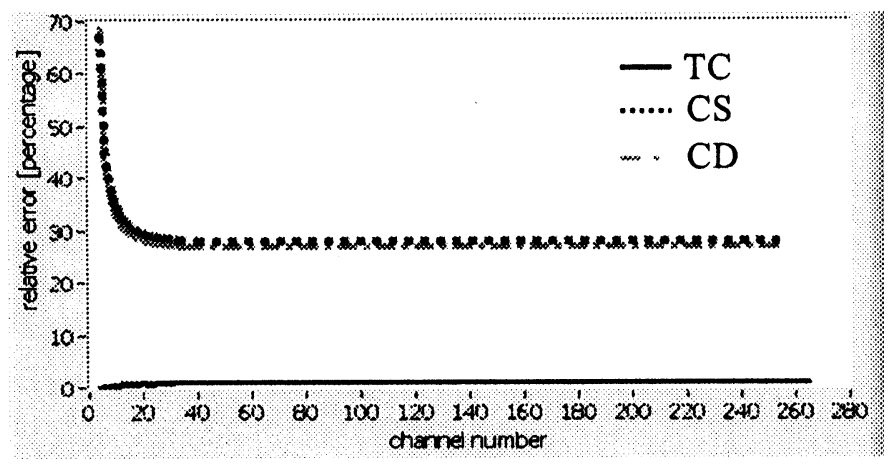

Fig. 5. Relative error $\varepsilon$ obtained with three different methods for the central comb channel versus the number of WDM channels in the case of SMF fiber.

which may reduce the accuracy of (3), depending on the vicinity of the ZDW to the WDM comb. The measured FWM power in this case is $\mathrm{P}_{f \mathrm{FWM} \mathrm{TC}}^{M}=\Sigma P_{i j k}\left(\eta^{\prime \prime}\right)$, where $\eta^{\prime \prime}$ is the efficiency evaluated by (3), which may differ from the actual efficiency $\eta$.

Figs. 4 and 5 show the relative error versus the number of WDM channels when measuring the FWM terms of the central comb channel at $\lambda_{\mathrm{c}}$, for a chromatic dispersion at $\lambda_{\mathrm{c}}$ equal to $2 \mathrm{ps} /(\mathrm{nm} \cdot \mathrm{km})(\mathrm{NZDSF})$ and $17 \mathrm{ps} /(\mathrm{nm} \cdot \mathrm{km})$ [standard single mode fiber (SMF)], respectively. The slope was $S=$ $0.04 \mathrm{ps} / \mathrm{nm}^{2} \cdot \mathrm{km}$, the channel spacing $0.4 \mathrm{~nm}$, and the WDM comb is symmetrically arranged around $\lambda_{c}$. The detuning in the $\mathrm{CD}$ method equals half the channel spacing. As seen, the proposed TC method allows for the measurement of the FWM crosstalk with a significantly better accuracy in both cases. The relative error which results is nearly independent on the chromatic dispersion value at $\lambda_{\mathrm{c}}$ and smaller than $2 \%$ for all channels, until the ZDW is not in the comb wavelength range. Otherwise, the accuracy in the FWM crosstalk prediction decreases, especially for the channels nearest to the ZDW.

\section{EXPERIMENTAL RESULTS}

To validate the TC method, we considered a four-channel system with spacing equal to $0.4 \mathrm{~nm}$. The measured

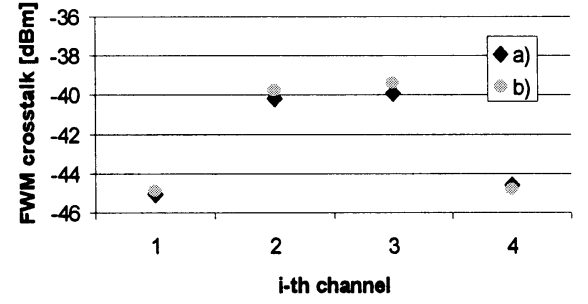

Fig. 6. FWM crosstalk for all channels of a four-channel system. a) (9) with TC method; b) (9) with measured fiber data.

fiber span parameters were chromatic dispersion = $-2.1 \mathrm{ps} / \mathrm{nm} \cdot \mathrm{km}$, nonlinear coefficient $=3.1 \cdot 10^{-20} \mathrm{~m}^{2}$, attenuation $=0.24 \mathrm{~dB} / \mathrm{km}$, length $=100.259 \mathrm{~km}$, and effective area $=55 \cdot 10^{-12} \mathrm{~m}^{2}$. With channels 3 and $4 \mathrm{OFF}$, we determined the efficiency $\eta_{1}$, and then with only channel 3 OFF we determined $\eta_{3}$, and thus the FWM crosstalk for each channel, as per (9). Finally, we compared the TC results with the FWM crosstalk values obtained from (9) when the efficiency is calculated from the measured fiber data. Fig. 6 shows the comparison. A good correspondence between the two calculations is obtained. The small discrepancies are mostly attributed to the limited accuracy in the measurement of fiber parameters, while clearly the TC method avoids the complexity of such measurements, and requires only two power measurements.

\section{CONCLUSION}

A novel method for FWM crosstalk measurement in DWDM has been presented. With special input two- and three-channel configurations, all the in-band FWM power contributions can be easily estimated by measuring the power of only two FWM terms. A comparison with other known techniques shows the higher precision of the present method.

\section{ACKNOWLEDGMENT}

A. Bogoni and L. Poti would like to thank Marconi Communications for helpful technical support.

\section{REFERENCES}

[1] R. W. Tkach, A. R. Chraplyvy, F. Forghieri, A. H. Gnauck, and R. M. Derosier, "Four-photon mixing and high-speed WDM systems," $J$. Lightwave Technol., vol. 13, pp. 841-849, May 1995.

[2] E. S. Georges, "Experimental study of four-wave mixing on dispersion shifted fiber and low dispersion fibers with a dense multiwavelength source," in Proc. OFC'97, 1997, pp. 67-69.

[3] E. Ciaramella, "Statistics of four-wave mixing impairments in nonuniform fiber links," in Prof. OFC'98, 1998, pp. 392-393. 